\title{
ACIDENTES, ENVENENAMENTOS E VIOLENCIAS COMO CAUSA DE MORTE DOS RESIDENTES NO MUNICIPIO DE SÃO PAULO, BRASIL
}

Silveira, M. II. \& Gotlieb, S. L. D. - Acidentes, envenenamentos e violencias como causa de morte dos residentes no município de São Paulo, Brasil. Rev. Saúde públ., S. Paulo, 10:45-55, 1976.

Resumo: Foi estudado o comportamento das mortes violentas de residentes no municipio de São Paulo, nos anos 1970, 1971 e 1972. Essas causas, que representam, aproximadamente, $10 \%$ do total de óbitos ocorridos em cada ano, incluem todos os tipos de acidentes (trânsito, doméstico, do trabalho e outros), os suicidios, homicidios e todas as demais causas externas. Os tipos de morte foram analisados segundo sexo e grupo etário, sendo os resultados apresentados através de mortalidade proporcional e do risco de morrer por essas causas.

Unitermos: Acidentes. Mortes violentas. Mortalidade.

\section{N T RODUCX O}

A importância das mortes violentas em geral, incluídas aqui aquelas por acidentes de todos os tipos, por homicídios e por suicídios, vem crescendo a cada dia. Seja nos países que já venceram a fase das moléstias infecciosas, seja mesmo naqueles em que estas figuram como uma das principais causas de morte, a magnitude do problema das mortes violentas assume destaque cada vez maior.

Do ponto de vista da saúde pública, é necessária uma análise do problema, visando a conhecer suas características, seu alcance e sua distribuição. Só recentemente, entretanto, é que se iniciou o estudo epidemiológico dos acidentes, pela crescente importância adquirida por esse grupo, tanto como causa de morte, como de incapacidade ${ }^{10}$.
As estatísticas mundiais têm mostrado que as taxas de mortalidade pelas causas violentas devem preferivelmente ser analisadas segundo seus componentes específicos, dado que o comportamento de cada um pode variar, dependendo do grau de desenvolvimento da região em apreço. Assim, por exemplo, na Finlândia, Hungria, Estados Unidos e Dinamarca, a representação do grupo de acidentes de veículos a motor e outros acidentes (principalmente de trabalho e domésticos) é bastante grande, chegando a valores aproximadamente iguais a 5,5 por dez mil habitantes $^{2}$ em anos próximos a 1970.

No Brasil, o número de óbitos pelas causas violentas, como um todo, é alto, devido à elevada ocorrência de acidentes de veículos a motor, outros acidentes e do

* Do Departamento de Epldemiologia da Faculdade de Saúde Pública da USP - Av. Dr. Arnaldo, 715 - São Paulo, SP, Brasil. 
SILVEIRA, M. H. \& GOTLIEB, S. L. D. - Acidentes, envenenamentos e violências como causa de morte dos residentes no municipio de são Paulo, Brasil. Rev. Saúde públ., S. Paulo, 10: $45-55,1976$.

grupo representado pelos homicídios, sendo, portanto, motivo de preocupação, dado que representa uma perda desnecessária de vidas ${ }^{9}$. Analisando os valores de algumas capitais brasileiras, verifica-se para o ano de 1971, em ordem decrescente, Recife com 9,95 por dez mil habitantes; Rio de Janeiro com 9,78; Salvador com 8,80; Brasília com 7,69; São Paulo com 6,92 e Porto Alegre com $6,20^{3}$. A posição ocupada por São Paulo, dentre essas capitais, revela sua situação precária e o problema se agrava ao se comparar com os dados mundiais apresentados. Em anos próximos a 1970 , aproximadamente $10 \%$ das mortes de pessoas residentes no município de São Paulo ocorreram devido ao grupo de causas relativo a acidentes, envenenamentos e violências.

Em 1970, para o sexo masculino, a primeira causa de óbito foi representada pelo grupo das doenças do coração (excluídas as reumáticas), que correspondeu a $16,83 \%$ do total de mortes. As violentas ocuparam o segundo lugar, com $12,55 \%$, sendo o terceiro posto representado pelo grupo de infecciosas, com $11,54 \%$. Em 1971 e 1972, para o mesmo sexo, a ordem das causas foi: cardíacas, infecciosas e acidentes, com valores respectivamente iguais a $16,16 \%, 12,35 \%$ e $11,87 \%$ para 1971 e $15,71 \%, 13,26 \%$ e $12,51 \%$ para 1972. Com relação ao sexo feminino, a localização percentual dos acidentes como um todo, não se encontra entre as cinco primeiras causas de morte, mostrando, que o problema se apresenta em magnitude diferente para os dois sexos.

O objetivo deste trabalho é descrever o comportamento dos acidentes, envenenamentos e violências na mortalidade geral dos residentes no município de São Paulo, no período de 1970 a 1972.

A análise desse comportamento pôde ser realizada através do estudo da mortalidade proporcional desse grupo de causas no total de óbitos e do risco de morte por essas mesmas causas. A mortalidade diferencial foi analisada segundo as variáveis sexo e idade.

\section{MATERIAL E MÉTODOS}

A população de estudo compreendeu todos os casos de óbitos por causas violentas de residentes no município de São Paulo, nos anos de 1970, 1971 e 1972, analisados segundo as variáveis citadas.

Os dados relativos a esses óbitos foram obtidos através das listagens do Departamento de Estatística da Secretaria de Economia e Planejamento do Estado de São Paulo (DEE)*.

Para causas violentas foram adotados os critérios da Classificação Internacional de Doenças (8. Revisão, 1965) ${ }^{8}$, onde se incluem as lesões recentes e os efeitos tardios dos acidentes, envenenamentos e outros tipos de violências. A Classificação utilizada foi a Lista B (Lista de 50 grupos de causas para tabulações de mortalidade), abrangendo os subgrupos BE-47, BE-48, BE-49 e BE-50, respectivamente:

BE-47: - Acidentes de veículos a motor, englobando acidentes de trânsito (E-810 a E-819) e os demais acidentes de veículos a motor (E-820 a E-823);

BE-48: - os demais acidentes:

- acidentes de estrada de ferro (E-800 a E-807);

- acidentes de outros veículos de estrada (E-825 a E-827);

- acidentes em transporte por água (E-830 a E-838);

- acidentes de transporte aéreo ou espacial (E-840 a E-845);

- envenenamentos acidentais por drogas e medicamentos (E-850 a E-859) ;

- envenenamentos acidentais por outras substâncias sólidas e líquidas (E-860 a E-869);

* Dados inéditos. 
SILVEIRA, M. H. \& GOTLIEB, S. L. D. - Acidentes, envenenamentos e violências como causa de morte dos residentes no municipio de São Paulo, Brasil. Rev. Saúde públ., S. Paulo, 10: 45-55, 1976 .

- envenenamentos acidentais por gases $\mathrm{e}$ vapores $(\mathrm{E}-870$ a E-877);

-- quedas acidentais (E-880 a E-887);

- acidentes causados pelo fogo e chamas (E-890 a E-899);

- acidentes devidos a fatores naturais e ambientais (E-900 a E-909);

- outros acidentes (E-910 a E-929);

- complicações e acidentes provocados por intervençōes cirúrgicas e médicas (E-930 a E-936);

- efeitos tardios das lesões provocadas por acidentes (E-940 a E-949).

BE-49: - Suicídios e lesões auto-inflingidas (E-950 a E-959);

BE-50: - Todas as demais causas externas, grupo que, para o fim deste trabalho, se apresenta dividido, seguindo a metodologia do D.E.E., em:

BE-50-A: homicídios e lesões provocadas intencionalmente por outras pessoas (E-960 a E-969), e
BE-50-B: intervenção le g a l (E-970 a E-978)

- lesões em que se ignora se foram acidental ou intencionalmente inflingidas (E-980 a E-989);

- lesões resultantes de operações de guerra (E-990 a E-999).

Para os cálculos de coeficientes de mortalidade por essas causas foram utilizadas as estimativas populacionais para $10^{\circ}$ de julho de 1970, 1971 e 1972, obtidas, pelo método geométrico, dos censos de $1960^{4}$ e $1970^{5}$. A composição das populações por sexo e idade seguiu a distribuição percentual apresentada pelo censo de $1970^{5}$

\section{RESULTADOS E DISCUSSAO}

\section{Mortalidade proporcional}

Para o sexo masculino, a mortalidade proporcional por causas violentas, dentre o total de óbitos foi de $13,06 \%, 11,87 \%$ e $12,51 \%$ respectivamente para 1970 , 1971 e 1972.

No total do grupo de causas violentas, cada subgrupo, conforme a lista $B$, foi representada pelos dados constantes da Tabela 1.

T A B E L A 1

Mortalidade proporcional para o sexo masculino segundo os subgrupos de causas violentas, no periodo 1970 a 1972 para os residentes no municipio de São Paulo.

\begin{tabular}{|c|c|c|c|c|}
\hline 7 & $\begin{array}{l}1970 \\
(\%)\end{array}$ & $\begin{array}{r}1971 \\
(\%)\end{array}$ & $\begin{array}{r}1972 \\
(\%)\end{array}$ & $\begin{array}{c}\text { Para o } \\
\text { triênio } \\
(\%)\end{array}$ \\
\hline \multicolumn{5}{|l|}{ Causas } \\
\hline Acidentes com veiculos a motor (BE-47) & 41,26 & 44,61 & 44,03 & 43,35 \\
\hline Outros acidentes $(B E-48)$ & 27,87 & 26,78 & 27,67 & 27,44 \\
\hline Suicídios $(\mathrm{BE}-49)$ & 9,99 & 9,76 & 8,26 & 9,29 \\
\hline Homicídios (BE-50-A) & 15,16 & 15,49 & 16,90 & 15,89 \\
\hline Demais causas externas (BE-50-B) & 5,72 & 3,36 & 3,14 & 4,03 \\
\hline Total & 100,00 & 100,00 & 100,00 & 100,00 \\
\hline
\end{tabular}


SILVEIRA, M. H. \& GOTLIEB, S. L. D. - Acidentes, envenenamentos e violências como causa de morte dos residentes no municipio de Sāo Paulo, Brasil. Rev. Saúde públ., S. Paulo, 10: $45-55,1976$.

Em relação ao sexo feminino, verificase que os valores representativos dos óbitos por causas violentas no total de óbitos desse sexo, foram, respectivamente, de $4,61 \%, 4,60 \%$ e $4,96 \%$ para os três anos considerados. Com o mesmo critério utilizado para o sexo masculino, a distribuição percentual de cada subgrupo $B$ no total de causas violentas, para o sexo feminino, está representada na Tabela 2 .

A análise das Tabelas 1 e 2 mostra a importância do subgrupo BE-47, representado pelos acidentes de veículos a motor, proporcionalmente às demais causas violentas, tanto no sexo masculino quanto no feminino.

Chama ainda a atenção o fato do número de óbitos por suicídio no sexo feminino apresentar-se em ascenção. Analisado como causa de morte dentre todas as ocorridas no sexo masculino e no feminino, é neste que atinge seu maior valor.

O homicidio, por sua vez, está crescendo para o sexo masculino e analisado, comparativamente, como causa de morte dentre todas as ocorridas em cada sexo, é entre os homens que se apresenta em níveis mais elevados.
Quando se leva em conta a idade para o estudo da mortalidade proporcional por causas violentas, a questão assume alguns aspectos característicos, que vale a pena serem ressaltados. Com essa finalidade foi construída a Tabela 3, que mostra a mortalidade proporcional média para os três anos considerados. Isso porque a análise de três anos seria insuficiente para revelar a tendência temporal assumida por esse grupo de causas.

A Tabela 3 evidencia, para o sexo masculino, que os grupos etários de 15 a 30 anos, que representam a parte mais jovem da faixa etária produtiva, têm exatamente nas causas violentas, seus principais motivos de óbito.

Assim, por exemplo, no grupo de 15 a 20 anos, $62,57 \%$ dos óbitos ocorrem por essas causas; no grupo de 20 a 25 anos o valor é de $63,43 \%$ e no grupo de 25 a 30 anos apresenta-se igual a $53,15 \%$.

Com relação ao sexo feminino, a mortalidade proporcional por essas causas, embora sempre com valores menores que para o sexo masculino, apresenta-se alta já na faixa etária de 10 a 15 anos $(30,28 \%)$. De 15 a 20 anos seu valor é de $39,72 \%$ e de 20 a 25 anos, $32,78 \%$.

T A B E L A 2

Mortalidade proporcional para o sexo feminino segundo os subgrupos de causas violentas, no período 1970 a 1972 para os residentes no município de São Paulo.

\begin{tabular}{|c|c|c|c|c|}
\hline $7+2$ & $\begin{array}{l}1970 \\
(\%)\end{array}$ & $\begin{array}{r}1971 \\
(\%)\end{array}$ & $\begin{array}{r}1972 \\
(\%)\end{array}$ & $\begin{array}{c}\text { Para o } \\
\text { triênio } \\
(\%)\end{array}$ \\
\hline Acidentes com veiculos a motor (BE-47) & 45,52 & 46,86 & 52,10 & 48,42 \\
\hline Outros acidentes (BE-48) & 26,57 & 28,14 & 23,03 & 25,74 \\
\hline Suicidios $(B E-49)$ & 12,22 & 13,53 & 14,17 & 13,37 \\
\hline Homicidios (BE-50-A) & 10,65 & 7,90 & 8,14 & 8,83 \\
\hline Demais causas externas (BE-50-B) & 5,04 & 3,57 & 2,56 & 3,64 \\
\hline Total & 100,00 & 100,00 & 100,00 & 100,00 \\
\hline
\end{tabular}




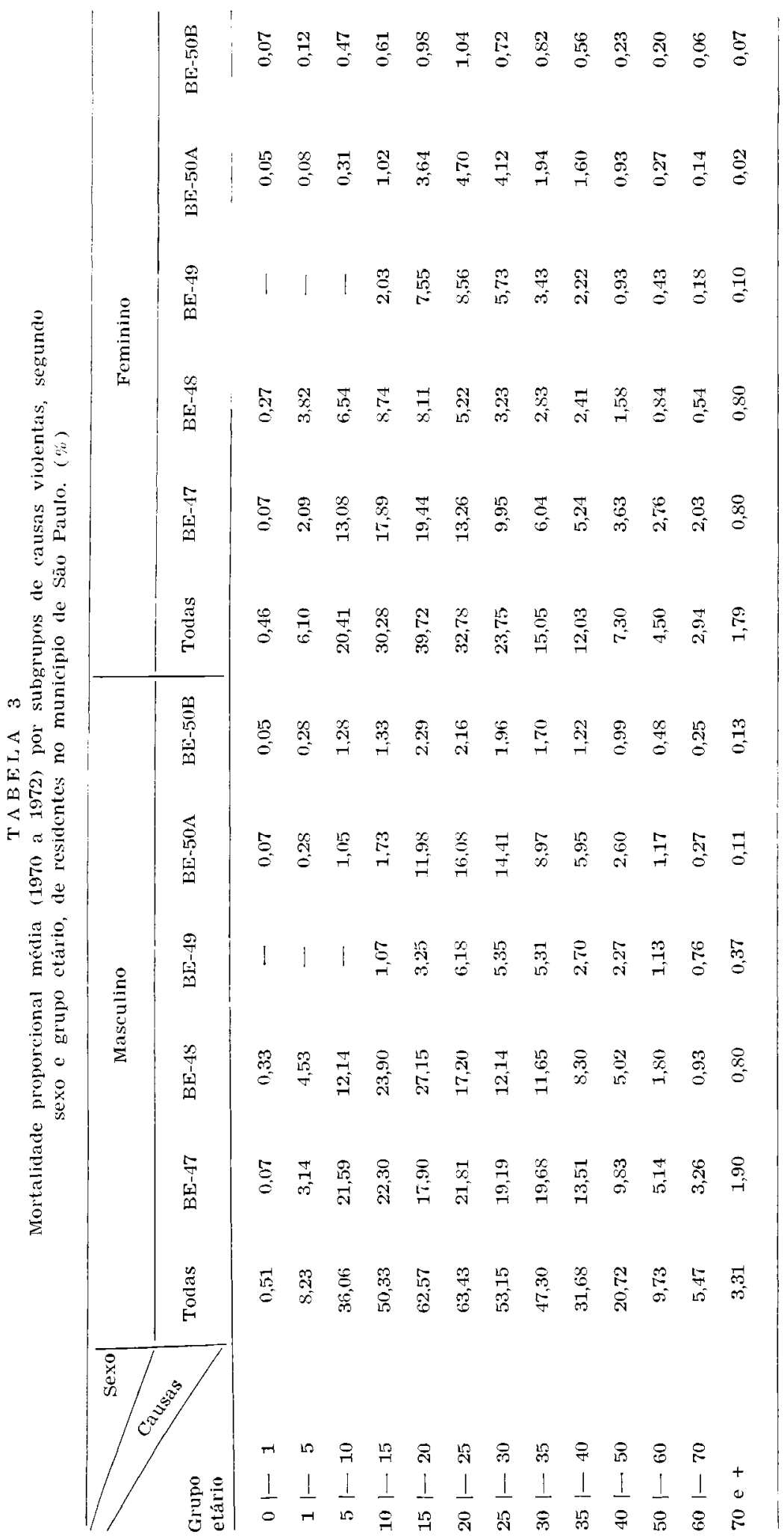


SILVEIRA, M. H. \& GOTLIEB, S. L. D. - Acidentes, envenenamentos e violências como causa de morte dos residentes no município de São Paulo, Brasíl. Rev. Saúde públ., S. Paulo, 10: 45-55, 1976.

Analisando o comportamento de cada um dos subgrupos B dentro de cada faixa etária, observa-se que, para o sexo masculino, o componente relativo aos acidentes de veículos a motor é, para o grupo etário acima de 20 anos, a mais importante das causas violentas. Para as idades de menos de 20 anos, o subgrupo BE-48 é o mais representativo, exceto na faixa 5 a 10 anos onde os acidentes de veículos a motor representam mais de $50 \%$ dos óbitos pelas causas violentas.

Entre as mulheres, apresentam-se também os acidentes de veículos a motor, proporcionalmente aos demais subgrupos $B$, como a causa mais representativa, em todas as idades acima de 5 anos. Abaixo desta faixa etária, é o subgrupo BE-48 a principal causa de morte, sendo que no grupo de 5 a 20 anos apareceu como a segunda causa de óbito.

Em relação ao suicídio, é na faixa de 20 a 25 anos, entre os homens, que apresenta seu maior valor, decrescendo nas faixas etárias seguintes. Para o sexo feminino, na idade de 20 a 30 anos, aparece o suicídio como a segunda causa mais importante.

No sexo masculino, no grupo de 15 a 30 anos, surge o homicídio como causa relevante, estando seu valor, aproximadamente, em torno de $15 \%$.

\section{Coeficientes de mortalidade}

$\mathrm{Na}$ análise dos coeficientes, procurou-se medir o risco de morrer pelas causas violentas, para cada sexo, nos anos de 1970, 1971 e 1972.

Os valores encontrados, para o sexo masculino, foram iguais a $11,19,11,05 \mathrm{e}$ 11,72 por dez mil habitantes, respectivamente para 1970, 1971 e 1972, sendo o coeficiente médio para o triênio, igual a 11,33 por dez mil habitantes. Pode-se concluir que o risco de morrer por esse grupo de causas, como um todo, mantevese mais ou menos constante para o período observado.

Com relação ao sexo feminino, os valores foram bem menores, o que revela a maior importância dessas causas entre os homens. Os coeficientes encontrados foram respectivamente $2,98,2,95$ e 3,35 por dez mil habitantes, para 1970, 1971 e 1972, sendo o valor médio, para o triênio, igual a 3,09 por dez mil habitantes.

Comparando os coeficientes segundo o sexo, verifica-se que o risco, no sexo masculino, é aproximadamente 3,5 vezes maior que no feminino, valor esse já encontrado por outros autores, em trabalho anterior ${ }^{6,7}$. Analisando este valor, somente com relação ao grupo dos acidentes de veículos a motor, poder-se-ia pensar numa maior exposição do sexo masculino ao risco de ter acidentes, dado que os homens se locomovem mais na cidade, principalmente pela mobilização ao trabalho ${ }^{6}$.

Estudando o risco de morte de cada um dos subgrupos B relativos às causas violentas, foram construídas as Tabelas 4 e 5, respectivamente para os sexos masculino e feminino.

Com referência ao sexo masculino, a Tabela 4 mostra a importância dos acidentes de veículos a motor (BE-47) como causa de morte. $O$ coeficiente vem se elevando nos três anos estudados, o mesmo ocorrendo com alguns dos outros subgrupos de causas violentas.

Apresenta-se em declínio o subgrupo dos suicídios, caindo também o subgrupo $\mathrm{BE}-50-\mathrm{B}$, representado pelas mortes em que se desconhece se foram ou não intencionalmente provocadas.

Com relação ao sexo feminino, chama a atenção na Tabela 5 , o fato do risco de morrer pelas causas do subgrupo BE-47, acidentes de veículos a motor, embora 
SILVEIRA, M. H. \& GOTLIEB, S. L. D. - Acidentes, envenenamentos e violéncias como causa de morte dos residentes no municipio de São Paulo, Brasil. Rev. Saúde públ., S. Paulo, 10: 45-55, 1976.

T A BELA 4

Coeficientes especificos de mortalidade, para o sexo masculino, segundo os subgrupos de causas violentas, no período de 1970 a 1972 , para residentes no munícíplo de São Paulo (por dez mil habitantes)

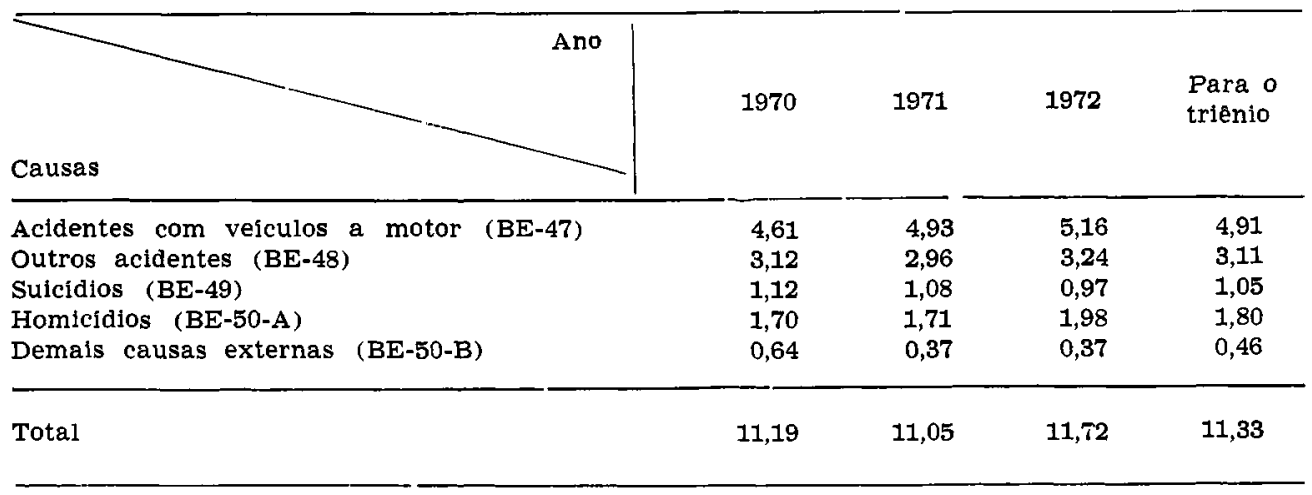

TABELA 5

Coeficientes especificos de mortalidade para o sexo feminino, segundo os subgrupos de causas violentas, no perfodo 1970 a 1972 , para residentes no município de são Paulo (por dez mil habitantes)

\begin{tabular}{l|llll}
\hline & 1970 & 1971 & 1972 & $\begin{array}{c}\text { Para o } \\
\text { triénio }\end{array}$ \\
Causas & & & & \\
\hline Acidentes com veículos a motor (BE-47) & 1,36 & 1,38 & 1,75 & 1,50 \\
Outros acidentes (BE-48) & 0,79 & 0,83 & 0,77 & 0,80 \\
Suicídios (BE-49) & 0,36 & 0,40 & 0,47 & 0,41 \\
Homicídios (BE-50-A) & 0,32 & 0,23 & 0,27 & 0,27 \\
Demais causas externas (BE-50-B) & 0,15 & 0,11 & 0,09 & 0,11 \\
\hline Total & 2,93 & 2,95 & 3,35 & 3,09 \\
\hline
\end{tabular}

muito menor que o correspondente ao sexo masculino, estar em ascensão, além de representar a mais importante dentre as causas violentas.

Com a finalidade de analisar os coeficientes médios - sexo masculino e feminino - apresentados nas Tabelas 4 e 5 e compará-los com os obtidos para o distrito de São Paulo em pesquisa anterior ${ }^{9}$, foi construído o gráfico da Figura, onde podem ser vistos os valores de cada subgrupo B, nos períodos estudados.

0 risco de morte por causas violentas, nos vários grupos etários, pode ser apre- ciado na Tabela 6, onde está retratado o coeficiente médio, para os dois sexos, no triênio examinado.

E importante ressaltar que o risco de morte por todas as causas violentas é maior no grupo etário de menores de um ano, que nos grupos etários seguintes, até os quinze anos para o sexo masculino e, surpreendentemente, até os cincoenta anos para o sexo feminnio. Em relação aos acidentes de veículos a motor, é crescente para os dois sexos, sendo, entretanto, sempre muito maior no sexo masculino. 
SILVEIRA, M. H. \& GOTLIEB, S. L. D. - Acidentes, envenenamentos e violèncias como causa de morte dos residentes no município de São Paulo, Brasil. Rev. Saúde públ., S. Paulo, 10: $45-55,1976$.

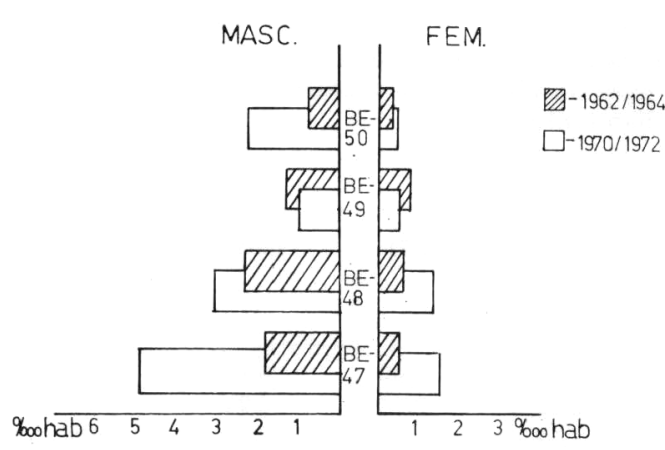

Figura - Coeficientes especificos de mortalidade ( $\tau_{0}$ o hab.) segundo subgrupos de causas violentas e sexo, em São Paulo, nos períodos $1962 / 1964$ e $1970 / 1972$.

Fonte: Puffer, R.R. \& Griffith, G.W."

Analisando o grupo de crianças menores de um ano, verifica-se a importância das causas englobadas no BE-48. Neste subgrupo, a principal causa foi a aspiração de alimentos que, em 1971, representou $38,46 \%$ do seu total. No mesmo ano, a sufocação mecânica e as quedas apresentaram-se como as causas seguintes, respectivamente $15,38 \%$ e $12,82 \%$.

No grupo 15 a 20 anos constata-se que o risco é bem diferente nos dois sexos, sendo muito maior no masculino que no feminino $(9,78$ e 2,49 por dez mil habitantes, respectivamente). Vale a pena salientar ainda que o BE-48 representado pelos outros acidentes, para o sexo masculino, é também maior que o BE-47, acidentes de veículos a motor, representando, aproximadamente, $50 \%$ dos óbitos pelas causas violentas. Uma possível explicação, visto que nessa categoria estão contidos os acidentes de trabalho, seria a de que, começando os jovens a trabalhar e dada talvez a sua inexperiência, estariam mais expostos ao risco de acidentarse. Ainda nesse grupo etário, verifica-se, para o sexo masculino, o aumento das causas BE-50-A, representadas pelos homicídios. Note-se que o que se quer salientar é que esta faixa etária se constitui no marco onde este problema - evidente- mente de caráter social - começa a aparecer.

De 20 anos em diante, são, indiscutivelmente, os acidentes de veículos a motor que assumem papel preponderante entre as causas violentas. Com referência ao homicídio, aumenta ainda mais, relativamente ao grupo etário anterior, atingindo seu maior valor na idade 25 a 30 anos, no sexo masculino, onde o risco se apresenta igual a 4.02 por dez mil habitantes.

É no grupo etário de 70 anos e mais, no sexo masculino, que ocorre o maior coeficiente, 30.6 por dez mil habitantes, do qual grande parte é reperesentada pelos acidentes de veículos a motor. Esse valor, entretanto, não significa necessariamente que haja maior número de acidentes com indivíduos dessa idade, mas talvez, dado que o acidente ocorra, que os indivíduos têm menor chance de sobreviver.

A análise do suicídio mostra que, muito embora a mortalidade proporcional por essa causa seja grande no sexo feminino, quando se analisa o risco de morrer por essa causa, apresenta-se ele maior entre os homens, vindo sempre num crescendo com o crescer da idade. Aliás, no mesmo sentido, conclui Barbosa ${ }^{1}$ em análise do problema relativamente aos anos de 1959 a 1968. No sexo feminino, o comportamento é diferente. Além do risco ser menor, a faixa etária de maior risco é a de 20 a 25 anos, decrescendo depois até a idade de setenta anos, quando de novo aumenta, como aliás também já demonstrara Barbosa ${ }^{1}$.

\section{CONCLUSOES}

1 - Os acidentes, envenenamentos e violências foram responsáveis por aproximadamente $10 \%$ das mortes de pessoas residentes no município de São Paulo, nos anos de 1970, 1971 e 1972;

2 - a mortalidade proporcional mostra a importância dos acidentes de veículos a motor, em relação às demais causas 


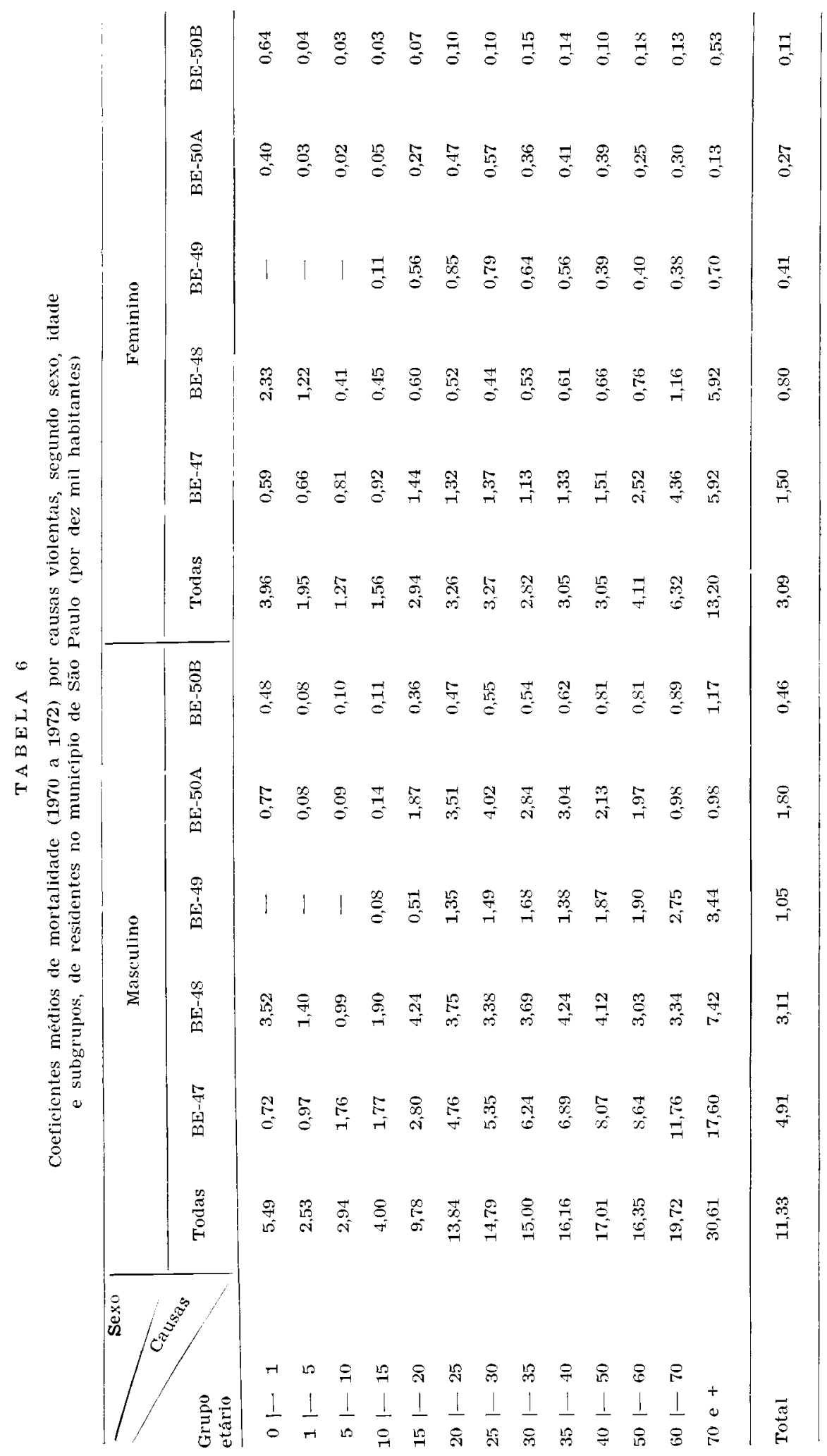


SILVEIRA, M. H. \& GOTLIEB, S. L. D. - Acidentes, envenenamentos e violências como causa de morte dos residentes no município de São Paulo, Brasil. Rev. Saúde públ., S. Paulo, 10: 45-55, 1976.

violentas. Para o triênio examinado, os valores foram de $43,35 \%$ e $48,42 \%$, respectivamente para os sexos masculino e feminino;

3 - o risco médio de morte por causas violentas foi igual a 11,33 por dez $\mathrm{mil}$ habitantes e 3,09 por dez mil habitantes, respectivamente, para os sexos masculino e feminino;

4 - o coeficiente específico de mortalidade por acidentes de veículos a motor vem se elevando, nos três anos estudados, tanto para o sexo masculino quanto para o sexo feminino; este risco é também crescente com a aumento da idade, em ambos os sexos;
5 - o risco de morte, por todas as causas violentas é maior no grupo etário de menores de um ano que nos grupos etários seguintes, até 15 anos no sexo masculino e até 50 anos no sexo feminino;

6 - na faixa de menores de um ano os acidentes domésticos assumem papel relevante, sendo o risco igual a 3,52 por dez mil habitantes entre os homens e 2,33 por dez mil habitantes no sexo feminino;

7 - o homicídio é importante no sexo masculino, a partir dos 15 anos, tendo seu maior risco na idade 25 a 30 anos;

8 - apesar da mortalidade proporsional por suicídio ser maior no sexo fernimino, o risco de morte é maior entre os homens.

Silveira, M. H. \& Gotlieb, S. L. D. - [Accidents, poisonings and violences as causes of death in resident inhabitants of the city of S. Paulo, Brazil]. Rev. Saúde públ., S. Paulo, 10:45-55, 1976.

SUMmaRY: The external causes of death for the residents of Sano Paulo, Brazil, are described according to mortality rates and ratios for the period 1970 to 1972. These causes of deaths (traffic, home and work accidents, homicides, suicides and all other) were responsible for $10 \%$ of the total deaths occurred in that period. The risk of death by external causes for all ages is 3,5 times higher for males (11,33\% inhab.) than for females (3,09\% inhab.). The death rate for motor vehicle accidents is increasing for both sexes in this period. The mortality rate for children less than one year old is very high and the main contribution was due to home accidents. Among the studied causes of death, homicide was a very important one for males aging 15 years old and over. The mortality ratio by suicide is higher for females than males but the risk of dying is higher for males.

UNITERMS: Accidents. Mortality.

\section{REFERENCIAS BIBLIOGRAFICAS}

1. BARBOSA, V. - Estudo descritivo do sulcídio no municipio de são Paulo (Brasil) de 1959 a 1968. Rev. Saíde puibl., S. Paulo, 8:1-14, 1974.

2. DEMOGRAPHIC YEARBOOK, 1973. (United Nations). New York, 1974.
3. FUNDAÇAO IBGE - Anuario estatistico do Brasil. Rio de Janeiro, 1974.

4. FUNDACXO IBGE - VIII Recenseamento geral. (Censo Demográfico, 1970). Rio de Janeiro, 1973. 
SILVEIRA, M. H. \& GOTLIEB, S. L. D. - Acidentes, envenenamentos e violências como causa de morte dos residentes no municipio de São Paulo, Brasil. Rev. Saúde públ., S. Paulo, 10: $45-55,1976$.

5. FUNDAÇÃO IBGE - Estado de Sĩo Paulo. Populaça presente na data do Censo, 1960 (Dados não publicados) (mimeografado).

6. LAURENTI, R. - Epidemiologia dos acidentes de trânsito. Ars Curand, S. Paulo, $7: 18-27,1975$.

i. LALRENTI, R. et al. - Alguns aspectos epidemiológicos da mortalidade por acidentes de trânsito de vejculo a motor na cidade de são Paulo, Brasil. Rev. Saude públ, S. Paulo, 6: $329-41,1972$.

8. ORGANIZAÇÃO PANAMERICANA DE SACDE - Manual de Classificaço
Estatistica Internacional de Doencas, Lesóes e Causas de óbito. 8. a revisão, 1965. Washington D.C., 1969. (Publicação científica n.o 190 ).

9. PUFFER, R R. \& GRIFFITH, G. W Caracteristicas de la mortalidad urbana. Washington D.C., Organización Panamerıcana de la Salud, 1968.

10. SAN MARTIN, H, - Salud y Enfermedad. 2.a ed Mexico. La Prensa Medica.

Recebido para publicacto em $08 / 12 / 1975$ Aprovado para mublicacio em oz/01/19\%6 